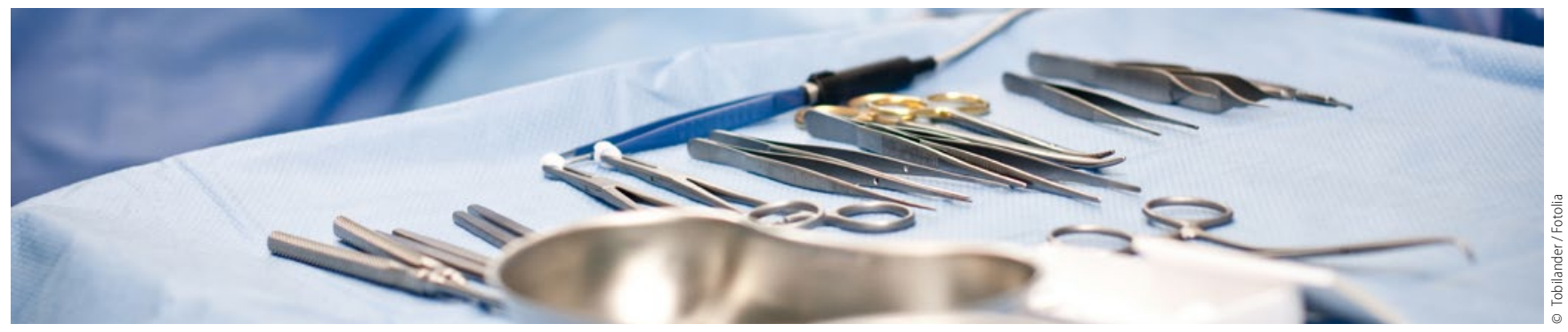

Das Surgery-First-Konzept und die Kortikotomie

\title{
Neue Aspekte der kieferorthopädischen Therapie
}

\begin{abstract}
Das Behandlungskonzept von Surgery First (Chirurgie zuerst) bezeichnet einen kombinierten kieferorthopädischen/kieferchirurgischen Therapieplan, der auf eine prächirurgische Behandlung durch den Kieferorthopäden komplett verzichtet. Die kieferorthopädische Therapie beginnt dabei erst kurze Zeit nach der chirurgischen Umstellung der Kiefer. Nahezu alle Patienten mit einer Malokklusion oder kraniofazialen Deformität können gemäß einer Studie der Universität Yale und einer Privatpraxis in Kolumbien durch eine Surgery-First-Therapie behandelt werden.
\end{abstract}

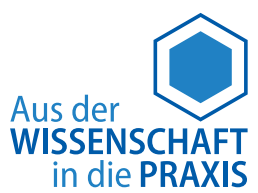

Als Vorteil werden die schnelle Auflösung der skeletalen Deformation, die einfachere Einstellung der Malokkusion bei fehlenden Weichgewebsinterferenzen und vor allem die kürzere Behandlungszeit genannt. Bislang wurde die kürzere Behandlungszeit als eine der Hauptargumente für das Surgery-First-Konzept vor allem in Fallberichten beschrieben, die Behandlungszeiten von weniger als ein Jahr angeben.

Die durchschnittliche Behandlungsdauer betrug in der vorliegenden Studie 10,5 Monate bei 13,8 Arztbesuchen. Mit dem konventionellen Konzept ist mit einer Gesamtbehandlungsdauer von 18 bis 36 Monaten zu rechnen. Verglichen mit der Surgery-First-Behandlung mussten sich die Patienten jedoch häufiger beim Kieferorthopäden vorstellen. In der vorliegenden Studie wurden die Qualität der Behandlung und die Stabilität des Ergebnisses allerdings nicht betrachtet.

\section{Wesentlich kürzere Behandlungs- und Heilungszeit}

Die kürzere Behandlungsdauer könnte möglicherweise auf ein aktiviertes „bone remodelling" nach einer Operation zurückgeführt werden, wie es von der Technik des ,corticotomy-assisted orthodontic treatment" (CAOT) bekannt ist. Das CAOT hat Möglichkeiten eröffnet, die kieferorthopädischen Behandlungszeit zu verkürzen, das alveoläre Knochenvolumen zu vergrößern, die Zähne präziser zu bewegen und zudem eine bessere Stabilität nach Abschluss der Behandlung zu gewähren. Damit kann die Behandlungszeit um das Drei- bis Vierfache reduziert werden. Der damit initiierte Heilungsprozess wird als „,regional acceleratory phenomenon" (RAP) bezeichnet. RAP war zunächst von Frost et al. (1983) beschrieben worden. Dieses Phänomen tritt bei jedem Trauma auf und beschleunigt die Heilung von Hart- wie auch Weichgewebe um das Zwei- bis Zehnfache. Die Heilung betrifft zum einen eine verminderte Knochendichte und zum anderen einen erhöhten Knochenumsatz (bone turnover). Die Kortikotomie wird seit Ende der 50er-Jahre zur Erleichterung der Zahnbewegungen eingesetzt. Kombiniert mit einer Applikation von partikulärem Knochenersatzmaterial wird diese Technik als ,periodontally accelerated osteogenic orthodontics" (PAOO) bezeichnet. Damit kann zum einen das alveoläre Knochenvolumen vergrößert und zum anderen die kieferorthopädische Behandlung beschleunigt werden.

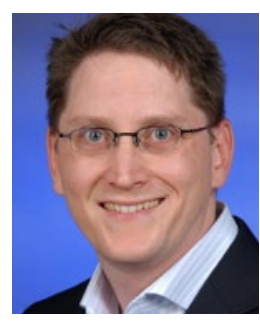

Prof. Dr. Dr. Felix P. Koch

Autor des Wissenschaftlichen Info-Dienstes, stellvertretender Klinikdirektor und Leitender Oberarzt für Mund-, Kiefer- und Gesichtschirurgie der Uniklinik Frankfurt/Main. 